\title{
Test de Rosa de Bengala en el diagnóstico diferencial del sindrome de ictericia obstructiva del primer trimestre
}

DRES. PATRICIO BRINCK M. ", JOSE ZACARJAS S. * e ISMAEL MENA G. **.

El sindrome de ictericia obstructiva del lactante menor es causado principalmente por las malformaciones de las vías biliares extrahepáticas y por las hepatitis neonatales.

El diagnóstico diferencial de estos dos cuadros clínicas ofrece frecuentemente grandes dificultades, en especial en etapas evolutivas avanzadas.

La observación clínica y el estudio seriado de laboratorio desde los primeros días de enfermedad pueden orientar hacia la etiología, pero con frecuencia deben analizarse en conjunto con la exploración quirúrgica, la colangiografía y la biopsia hepática para poder llegar a un diagnóstico certero.

En los casos de atresia de las vías biliares se observa alteración cirrótica ya desde el $2^{\circ}$ mes de vida, por lo que cualquier intento de corrección quirúrgica debe ser realizada antes de esa edad.

En la búsqueda de nuevos métodos diagnósticos, que evitaran el riesgo de la laparotomía exploradora a las hepatitis neonatales, Brent y Geppert en 1957 utilizaron con éxito una modificación del test de Rosa de Bengala marcado con I 131 introducido en adultos por Taplin en 1955.

La presente comunicación tiene por objeto dar a conocer los resultados obtenidos con este medio diagnóstico, que utilizamos desde 1967.

Material Y métódos. 1. El test de Rosa de Bengala. El Rosa de Bengala es un colorante de fluoresceina yodado que inyectado por vía endovenosa es captado exclusivamente por el hepatocito y luego excretado rápidamente por la bilis al intestino desde donde no sería reabsorbido. (fig. 1).

\footnotetext{
* Servicio de Pedialria, Hospital Calvo Mackenna, Santiago,

* Servicio de Medicina Nuclear, Hospital Clínico de la Universidat Católica de Chíle, Santiago, Chise.
}

Por estas propiedades se utilizó a partir de 1924 en la evaluación de la función hepática midiendo el porcentaje de retención sanguínea.

Posteriormente en 1955 Taplin reemplazó en la molécula el yodo por yodo 131 permitiendo su detección por conteo en el organismo y en las excretas y su utilización en el estudio de la permeabilidad de las vías biliares. En la obstrucción total de éstas sólo se encuentran cantidades muy pequeñas de I 131 en deposiciones que llegan por vía hematógena una vez disociado del Rosa de Bengala retenido en el hígado. La mayoría de este I 131 libre es captado por el tiroides y el resto eliminado por la orina. Con el propósito de evitar un eventual daño de esta glándula se Ja bloquea administrando 3 gotas de Lugol 2 veces al día desde 24 horas antes de la inyección del medio.

La cantidad exacta del radioisótopo utilizado se midió previamente en un contador de pozo. Se inyectó Rosa de Bengala con 20 microcuries de I 131 por vía endovenosa, cuidando especialmente el evitar su caída al tejido subcutáneo.

Posteriormente se midió la radiactividad residual en los materiales utilizados, como jeringas, agujas y algodones, restándola de la cantidad inicial. Así se pudo determinar exactamente el isótopo inyectado en el torrente circulatorio.

Luego se colocó a estos pacientes en camas metabólicas con el propósito de recolectar las excretas durante 48 hrs., evitando cuidadosamente la contaminación con orina.

El contenido de Rosa de Bengala I 131 en estas deposiciones fue determinado por un detector de centelleo expresándose como porcentaje de la dosis administrativa por vía endovenosa.

2. Material clínico. Se estudio un total de 25 nińos: 13 casos de hepatitis neonatal, 10 casos de atresias de las vías biliares extrahepáticas, y 2 


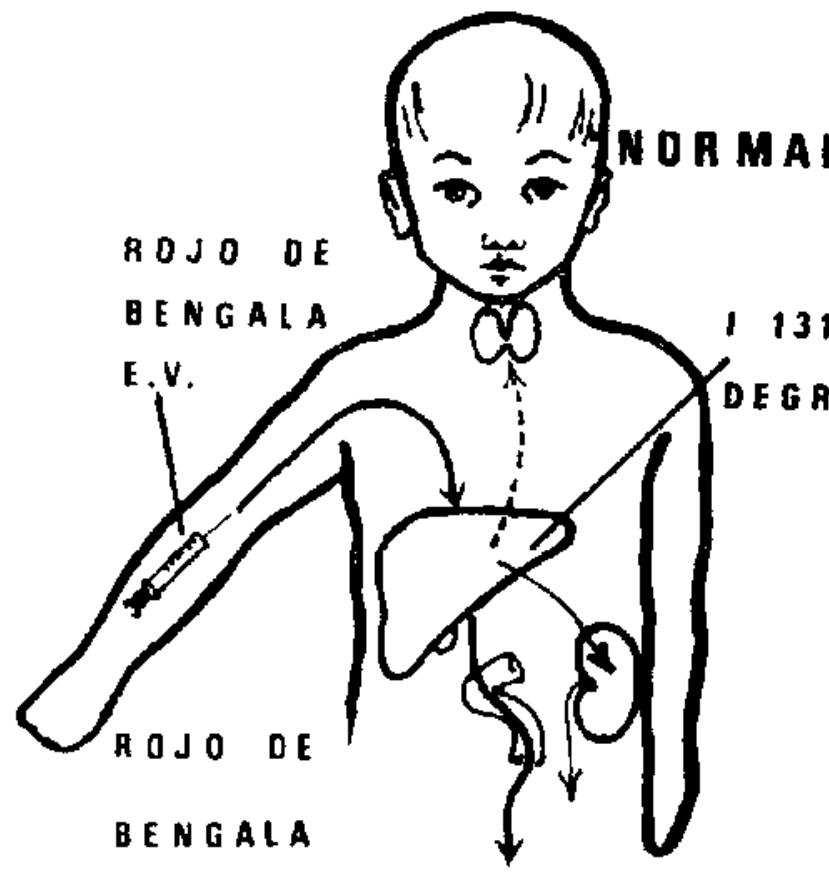

EN OEPOSICIOHES

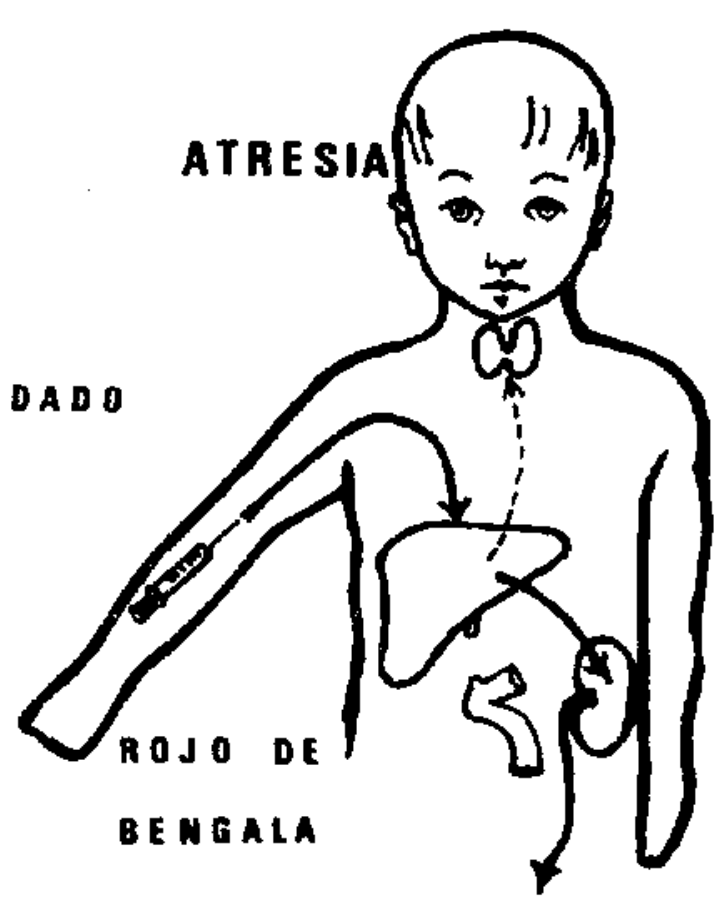

EN OAINA

Fig. 1.- Vias de metabolización del kase de Bcngala $I$ te 131 en el sujeto nornal y en el portador alc una áresia de vías biliares extrahepáticas.

casos de ictericias obstructivas no corresponjientes al primer semestre de la vida, una atresia jntrahepática en un niño de 3 años 9 meses y una probab e cirrosis biliar primaria en una niña de 2 años 6 meses.

No se realizó el test de Rosa de Bengala en sujctos controles sancs por considerarse innecesario.

A todos los enfermos se les efectuó un comfieto estudio de laboratorio junto con una obser váción clínico acuciosa.

De $\operatorname{los} 13$ casos de hepatitis neonatales 6 fucrin explorados quirúrgicamente, practicándoseles c) langiografía intraoperatoria directa y biopsia hepática. Sólo en un caso se hizo biopsia por punción.

Los 6 casos restantes poseían suficientes elementos c:ínicos y de laboratorio como para poder descartar una atresia sin necesidad de practicar laparatemia. La evolución posterior con recuperación total de 5 niños, y muerte por cirrosis postnecrót:ca con vía biliar normal en el otro, vino a confirmar esta actitud.

A este út timo caso citado, fallecido a los $5 \mathrm{mc}-$ ses 24 dias. se suma el de otro paciente, en el cual sa comprobó una hepatitis neonatal por la biopsia hepática quirúrgica, que falleció en el postoperatorio a los 6 meses 12 días por periton:tis secundaria a un absceso del hilio hepático.

De los 10 casos de atresias de las vías biliares cxtrahepát'cas, 9 fueron explorados quirúrgicamente en una edad promedio de 85 días. La intervención más precoz fue a los 40 días y la más tardía a los 120 días. El décimo fue operado a los 7 meses 28 días.

En todos se comprobó atresia de todas las vias biliares extrahepáticas. Sólo en un caso existía una pequeña vesícula cística sin bilis, pero con cístico, colédoco y hepático atrésicos. En éste se intentó una colangiografía itraoperatoria y se efectuó biopsia hepática que comprobó una atresia.

Ninguno de los casos fue considerado susceptible de corrección quirúrgica.

4 de estos pacientes no volvieron a control ignorándose su evolución posterior. 5 fallecieron con una sobrevida máxima de 10 meses 18 días.

Sólo en uno de estos niños se efectuó necrops'a ya que el resto falleció en su domicilio. En ese caso se pudo comprobar la atresia completa.

Un caso continúa vivo al año nueve meses de vida, con cirrosis franca con hipertensión portal y $41 \mathrm{mg}$.\% de bilirrubina total. 
TEST DE ROJO DE OENGALA J"132

EN EL SINDAOME OE ICTERICIA OBSTRUCTIVA DEL PAIMER

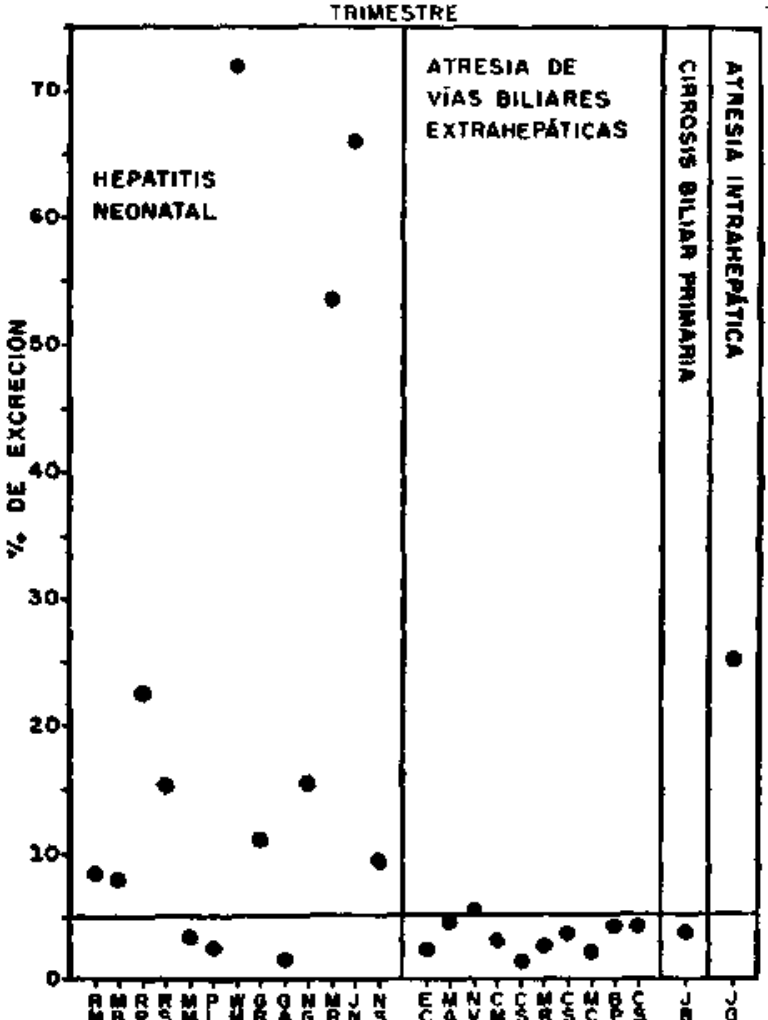

Fig. 2, - Resultados obtenidos en 25 nin̄os en los que se praelicó el lést de Rosa de Bengala I+ 131 .

Resultados. Los resultados del test de Rosa de Bengala efectuado se observan en la figura 2 .

En el grupo formado por 13 pacientes con hepatitis neonatal obtuvimos cifras que variaron en un amplio margen.

El porcentaje más elevado fuc de $71,9 \%$ similar al encontrado como normal en controles sanos en otras experiencias.

El más bajo fue de 1,6\% correspondiente a un niño que, controlado hasta ahora $\mathrm{y}$ teniendo actualmente 4 años, se ha recuperado en forma completa. En él, tanto la exploración quirúrgica como la colangiografía operatoria revelaron una vía biliar normal.

Otros 2 enfermos mostraron porcentajes bajos de excreción fecal: uno, $3,3 \%$ también completamente recuperado y con vía biliar normal, y otro, con $2,5 \%$ portador de una cardiopatía congénita acianótica, en el cual la colangiografía reveló paso del medio al duodeno. De este último enfermo no poseemos datos de su evolución más allá de los 3 meses.

Todos los resultados obtenidos en los casos de atresias fueron inferiores al $6 \%$. El más alto fue de $5,3 \%$ y el más bajo de $1,37 \%$ con un promedio de $3,1 \%$.

EI caso considerado como una probable ci- rrosis biliarr primaria dio un porcentaje bajo: de un $3,6 \%$ de excreción.

El enfermo portador de una atresia intrahepática, condición comprobada por el estudio histológico excretó un $25,07 \%$ del Rosa de Bengala.

Comentario. Los resultados obtenidos en esta experiencia son semejantes a los publicados por otros autores (Brent) (Ghadimi) (Sharp).

Se puede concluir que, valores sobre un $6 \%$ de excreción fecal de Rosa de Bengala permiten desechar el diagnóstico de una atresia de vías biliares. En cambio, bajo un $6 \%$ no se pueden eliminar completamente las hepatitis neonatales.

Este examen tiene por lo tanto indudable utilidad para seleccionar los pacientes sospechosos de atresia de las vías biliares que deben ser so. metidos a exploración quirúrgica, evitando así riesgos innecesarios a muchos casos de hepatitis neonatal.

La técnica de este examen es relativamente fácil, pero presenta varias posibilidades de error que deben ser considerados. Desde luego se tiene que disponer de un laboratorio de radioisótopos, los que no son muchos en nuestro medio.

La inyección endovenosa es difícil en lactantes pequeños, pudiendo quedar alguna cantidad del medio en el tejido subcutáneo, lo que evidentemente altera el resultado final.

La recolección de deposiciones sin contaminación con orina, es el principal problema, especialmente cn los enfermos del sexo femenino. Al estar bloqueada la eliminación por la vía biliar, el I 131, desdoblado del Rosa de Bengala en el hígado es eliminado por la orina, bastando que pequeñas cantidades de ésta contaminen las deposiciones para falsear el conteo final.

Finalmente, la constipación, frecuente en estos enfermos, puede ser otra causa que invalide el test.

Hasta ahora se aceptaba que no existiría rcabsorción intestinal del Rosa de Bengala excretado por el hígado, pero últimamente ha sido descrito un cierto grado de reabsorción, formándose un circuito enterohepático. Esto explicaría los valores bajos de excreción en las hepatitis neonatales.

La administración de resinas de intercambio aniónico, como la colesteramina, evitaría esta reabsorción, elevando el porcentaje de excreción fecal cuando no hay obstrucción biliar completa. Esta modificación no ha sido utilizada por noso. tros.

Debemos insistir que, la mayor seguridad para obtener un diagnóstico final certero, lo da sólo el análisis precoz y periódico de los hallazgos clinicos, exámenes de laboratorios $\mathrm{y}$ finalmente la exploración quirúrgica con colangiografía intraoperatoria y biopsia hepática.

Todas estas técnicas de estudio junto con las tcrapéuticas médico-quirúrgicas, son difíciles y cs- 
pecializadas, por lo que es muy importante trabajar en equipos que centralicen este tipo de pacientes.

\section{RESUMEN}

1.- Se presenta la experiencia en el test de Rosa de Bengala en 25 niños portadores del sindrome de ictericia obstructiva del primer trimestre.

2.- Se concluye que valores sobre un $6 \%$ de excreción fecal de Rosa de Bengala permiten descartar el diagnóstico de una atresia de vías biliares. Esta cifra es similar a la publicada por otros autores.

3.- Se aconseja utilizar este método en la selección de los enfermos cuya vía biliar deba ser explorada quirurgicamente.

\section{SUMMARY}

1.- The Rose Bengal I 13 | test was carried out in 25 infants with protracted obstructive jaundice in the first 3 months of age.

2. $\rightarrow$ Our findings, similar to those published by other althors, suggest that excretion of more than $6 \%$ of the rose bengal $I+131$ in the stools makes very improbable the diagnosis of biliary atresia.

3.- We advice the use of this method in the selection of those patients who should undergo a surgical exploration of the biliary tract.

\section{Bibliografía}

1.-Delprat, G, D., Epstein, N. N., and Kerr, W. J.: New liver function test: elimination of rose bengal when inyected into circulation of human subjects. Arch. Int. Med. 34: 533-541, 1924.

2.-Kerr, W. J., Delprat, G. D., Epstein, N. N., and Dunievirz, M.: Rose Bengal test for liver function: studies on rate of elimination from circulation in man. J.A.M.A. 85: 942-946, 1925.

3.-Taplin, G. V., Mereditm, O. M., Jr, und Kade, M.: Radioactive (I 131 - tagged) rose bengal uptake excretion test for liver function using external gamma-ray scintillation counling techniques. J. Lab. and Clin Med. 45: 665-678, 1955.
4.-Nordyke, R. A., and Blahd, W. H.: Differential diagosis of biliary tract obstruction with radioactive rose bengal $\mathrm{J}$. Lab, and Clin. Med. 51: 565$579,1958$.

5.-Geppert, L. J., and Brent R. L.: Radioactive rose bengal: aid in differential diagnosis of jaundice infant. J. Dis. Chil., 94; 544, 1957.

6.-Brem, R. L., and Geppert, L. J.: Use of radioactive rose bengal in evaluation of infantile jaundice. $J$. Dis. Child. 98: 720.730, 1959.

7.-Ghadimi, M., and Sass-Kortsak, A,: Evaluation of Radioactive Rose Bengal test for the differential Diagnosis of Obstructive Jaundice in Infants, New Eng. J. Méd. 265: 351, 1961.

8.-Sharp, H. L., Krivit, N., and Looman, J. T.: The Diagnosis of complete Extra hepatic Obstruction by Rose Bengal J. Pediat. 70: 46, 1967.

9.-Hays D. M., Woolley M. M., Snyder W. H., Reed G. B.: Diagnosis of Biliary Atresia: Relative Accuracy of Percutaneos Liver Biopsy, Open Liver Biopsy and Operative Cbolangiography. J. Pediat. 71 : 598-607, 1967.

10,- Thater M. M., and Gellis S. S.: Studies in Neonatal Hepatitis and Biliary Atresia: II. The effect of diagnostic laparatomy en long-term prognosis of neonatal hepatitis. Amer. J. Dis. Child., 116; 262, 1968.

11.-Mena L., Maggiolo C., Ríos E., y Badia W.: Determinación de volutrten sanguíneo con albúmina yodo 131. Rev. Med. de Chile, 90: 881-886, 1962.

12.-Poley J. R., Smith E. I, Boon D. J., Smith C. W.: Radioionated Rose Bengal and oral cholestyramine: identification of patent extrahepatic biliary pathways. The American Pediatric Society, Inc. and The Society for Pediatric Research. Program and Abstracts. April 1971.

\section{RECONOCIMIENTO}

So agradece la valiosa colaboración prestada en esta investigación pur el Departarnento de Cirugla y Ortopedia Infantil del Huspilal Calvo Mackenna de Santiago. 